\title{
Medical Debates and Medical Reversal
}

\author{
Adam S. Cifu, $\mathrm{MD}^{7}$ and Vinay K. Prasad, MD, MPH ${ }^{2}$ \\ 'University of Chicago, Chicago, IL, USA; ${ }^{2}$ Oregon Health and Sciences University, Portland, OR, USA.
}

$\mathrm{J}$ Gen Intern Med 30(12):1729-30

DOI: $10.1007 / \mathrm{s} 11606-015-3481-5$

() Society of General Internal Medicine 2015

$\mathrm{M}$ edical reversal occurs when an accepted practice- a diagnostic test, medication, or procedure-is overturned. The practice is not replaced by something better, but shown to be inferior to a preexisting, less intensive, or less invasive one. Sometimes the practice that is overturned is shown to be inferior to no intervention. Every physician is aware of emblematic cases of reversal, such as the use of hormone therapy in post-menopausal women. Medical reversal is common; well over 100 studies have been published in major medical journals over the last 10 years that provide strong evidence that an accepted medical practice was ineffective or harmful. ${ }^{1,2}$ The debates that follow these publications - whether the reversal concerns a screening test, surgical procedure, medical therapy, or systems intervention - are among the most contentious in medicine. These arguments are important ones; they ultimately decide how we, as a profession, progress in our effort to provide highvalue care, and they articulate how we view the standards of evidence upon which we base our practice. The debates that follow reversals adhere to a predictable script, pitting minimalists against maximalists. These debates also place the practicing physician, who must decide what to actually recommend to patients, in a difficult position.

Medical reversal occurs when practices are adopted before robust data is obtained. Even in our age of evidence-based medicine, this occurs frequently. A practice might be adopted because it makes excellent physiologic, biochemical, or molecular sense. It might be supported by observational trials. Interventions proven in severe or late-stage disease are sometimes used for less severe or preclinical disease, and data supporting care in young patients with few comorbidities may be extrapolated to elderly patients with coexisting diseases. Occasionally, well-done randomized trials find that a practice, adopted based on one of these less than robust forms of evidence, does not improve meaningful health outcomes. While there are examples where one of the above root causes predominates (for instance, interventional therapy for renal artery stenosis makes sound pathophysiologic sense, estrogen replacement was supported by robust observational trials, and SSRIs were proven effective in severe depression), most

Published online July 30, 2015 practices destined for reversal draw upon all of the weaker forms of evidence above.

When a large, well-powered randomized trial suggests that a practice be abandoned, the debate begins. Deimplementation does not happen rapidly, ${ }^{3}$ and recent work suggests that the forces against abandonment are predictable. ${ }^{2}$ Commercial entities and specialty societies seem particularly unlikely to embrace data that casts doubt on accepted therapies. ${ }^{2}$

The ongoing debate about the value of mammography is typical of those that follow medical reversal. Evidence suggests that mammography reduces the risk of dying from breast cancer, but its effect on overall mortality is uncertain. ${ }^{4}$ If mammography does decrease overall mortality, the magnitude of this benefit is so small that it would require enormous studies to reveal it. Mammography is also associated with false-positive test results that cause real harm via anxiety and overdiagnosis. ${ }^{5}$

Those who argue that a recent negative mammography trial $^{6}$ should put an end to the use of mammogram-based breast cancer screening contend that the randomized controlled trial is our best source of data. These "minimalists" cite the evidence of harm (overdiagnosis and overtreatment). They also note the lack of any robust data supporting mammography. They discount population data showing a small decrease in advanced breast cancer in the age of screening and argue that the promise of benefit associated with early detection is a false one without mortality data. On the other hand, "maximalists" contend that current breast cancer screening should continue despite the negative data. They argue that the studies we have are underpowered and use outdated imaging technology. Furthermore, they argue that abandoning screening for this potentially fatal disease is abrogating our responsibility to do the best for patients.

These arguments are predictable and analogous to those made regarding reversed practices as diverse as PCI for stable coronary disease, arthroscopic knee surgery for degenerative meniscal tears, and prostate cancer screening. Each side's argument contains truth and overstatement. Minimalists argue that, based on the newest research, the current practice is unwarranted. They argue that less can be more in health care and that our quest for high-value care necessitates abandoning interventions that cannot be clearly shown to benefit patients with regard to important clinical endpoints - mortality and quality of life. This group often completely discounts the less robust data that came before the most recent randomized trials. At their most extreme, they ignore the possibility that the 
Table 1 Counseling Patients on the Continued Use of a Practice after its Reversal

\section{Type of patient}

Data supporting the practice prior to reversal

Convincing pathophysiology

Case series

Observational trial

Randomized trial with surrogate endpoint

Single-center or small RCT

\section{Patients with a disease}

Practice only offered within trial

Practice only offered within trial

Intervention offered after review of current evidence

Practice offered after review of current evidence

Practice offered after review of current evidence
Healthy patients

Practice only offered within trial

Practice only offered within trial

Practice only offered within trial

Practice only offered within trial

Practice offered after review of current evidence treatment has small benefits, not detected in randomized trials, or that it benefits a subset of patients. They may be more apt to advise completely abandoning a treatment rather than collecting data in a more select population.

Maximalists argue that the current practice remains valuable in spite of robust data to the contrary. They usually identify flaws in the new data. They argue that the negative studies are under-powered, that they include problematic populations or study an intervention that is out is date. These arguments are imperfect in that they demand proof of ineffectiveness rather than proof of benefit. The argument that the absence of evidence is not evidence of absence is especially weak when it is used to justify actively deploying a medical intervention on a large scale. It runs directly against our mandate to "first, do no harm."

Left in a difficult position during these debates are the physicians who care for patients. The recommendation for them is usually to carefully inform their patients about both sides of the argument. Anyone who has had these conversations with patients knows well how they turn out. A small number of "minimalist" or "maximalist" patients reject or accept the treatment with little consideration. Most patients end up as confused by the conflicting data as we physicians are, and defer to our (presumed) expertise.

We need to elevate the debate about reversed practices and move away from black and white arguments. Although, as the maximalists argue, a modified version of a contradicted practice - or the practice employed in a subgroup of patients - might be effective, this is a hypothesis to be prospectively studied rather than an assumption to be accepted. The minimalists must be open to the possibility that future prospective studies could demonstrate that the contradicted practice has a place in medicine. Even a well-done randomized trial is not the final word on a practice. Our statistics include accepted error rates, a single trial only studies a single population, and many clinical trials are, in fact, outdated at the time of their publication.

As is usually the case, the practicing physician has the hardest job. Whether a practice is still recommended after a trial suggests that it is ineffective depends on many factors.
These include the strength of the data that initially supported the practice and whether the practice is a therapeutic or preventive one. Practices that are least likely to be effective should probably be offered only within clinical trials, while those more likely to provide benefit might be offered after more extensive counseling regarding the current state of the evidence. Table 1 suggests one way to consider this situation.

Medical reversal is not uncommon. It leads to debate within the field and controversy regarding how we treat our patients. As physicians, we must commit to proving that our interventions accomplish what we believe they do, rather than assuming that every new therapy inevitably leads to a better outcome. We must also acknowledge that not every therapeutic decision we make will be based on perfectly designed randomized controlled trials. While we should aim to adopt only therapies that are proven, we should also respond to the inevitable setbacks with thoughtful debate and careful counsel for our patients.

Conflict of interest: The authors declare no conflict of interest.

Corresponding Author: Adam S. Cifu, MD; University of Chicago, 5747 S. Dorchester Ave., Chicago, IL 60637, USA (e-mail: adamcifu@uchicago.edu).

\section{REFERENCES}

1. Prasad V, Vandross A, Toomey C, et al. A decade of reversal: an analysis of 146 contradicted medical practices. Mayo Clin Proc. 2013;88:790-8.

2. Wang MM, Gamble G, Grey A. Responses of specialist societies to evidence for reversal of practice. JAMA Intern Med. 2015.

3. Prasad V, Ioannidis JP. Evidence-based de-implementation for contradicted, unproven, and aspiring healthcare practices. Implement Sci. 2014;9:1.

4. Biller-Andorno N, Juni P. Abolishing mammography screening programs? A view from the Swiss Medical Board. N Engl J Med. 2014;370:1965-7.

5. Bleyer A, Welch HG. Effect of Three Decades of Screening Mammography on Breast-Cancer Incidence. N Engl J Med. 2012;367:1998-2005.

6. Miller AB, Wall C, Baines CJ, et al. Twenty five year follow-up for breast cancer incidence and mortality of the Canadian National Breast Screening Study: randomised screening trial. BMJ. 2014;348:g366. 